\title{
Modeling of the Transient Ni-like Ag X-Ray Laser
}

\author{
R.F.Smith, D. Benredjem, C. Moller, J. Kuba, A. Klisnick, \\ G.J. Pert, L. Upcraft, R. King, J. Gauthier, and L. Drska
}

This article was submitted to the International Society for Optical Engineering (SPIE) Meeting, San Diego, CA July 29 - August 3, 2001

February 26, 2002

U.S. Department of Energy

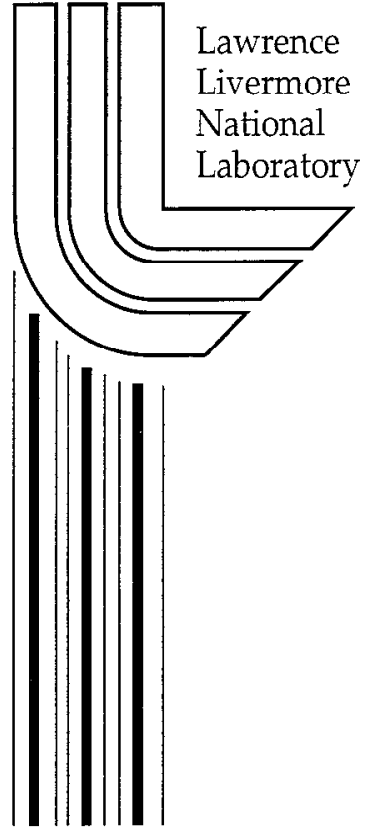




\section{DISCLAIMER}

This document was prepared as an account of work sponsored by an agency of the United States Government. Neither the United States Government nor the University of California nor any of their employees, makes any warranty, express or implied, or assumes any legal liability or responsibility for the accuracy, completeness, or usefulness of any information, apparatus, product, or process disclosed, or represents that its use would not infringe privately owned rights. Reference herein to any specific commercial product, process, or service by trade name, trademark, manufacturer, or otherwise, does not necessarily constitute or imply its endorsement, recommendation, or favoring by the Uniled States Government or the University of California. The views and opinions of authors expressed herein do not necessarily state or reflect those of the United States Government or the University of California, and shall not be used for advertising or product endorsement purposes.

This is a preprint of a paper intended for publication in a journal or proceedings. Since changes may be made before publication, this preprint is made available with the understanding that it will not be cited or reproduced without the permission of the author. 


\title{
Modeling of the transient Ni-like Ag X-ray Laser
}

\author{
Raymond F. Smith ${ }^{1 \text { }}$, Djamel Benredjem ${ }^{1}$, Clary Möller ${ }^{1}$, Jaroslav Kuba ${ }^{1,4}$, \\ Annie Klisnick', Geoffery J. Pert ${ }^{3}$, Lee Upcraft ${ }^{3}$, Robert King ${ }^{3}$, Jean-Claude Gauthier ${ }^{2}$, \\ Ladislav Drska ${ }^{4}$ \\ ${ }^{1}$ LSAI, Bat 350, Universite Paris-Sud, Orsay 91405, France. \\ ${ }^{2}$ LULI, UMR 3705 CNRS/CEA/Universite Paris VI, Ecole Polytechnique, 91123 \\ Palaiseau, France. \\ ${ }^{3}$ Department of Physics, University of York, Heslington, YO10 6DD, York, UK. \\ ${ }^{4}$ Faculty of Nuclear Sciences and Physical Engineering, Czech T'echnical University, \\ Brehova 7, 11519 Prague 1, Czech Republic.
}

\begin{abstract}
Recent high temporal resolution Ni-like $\mathrm{x}$-ray laser experiments have yielded important insights into the output characteristics of picosecond pumped x-ray lasers. However, current experimental observations do not fully explain the plasma dynamics which are critical to the gain generation within the $\mathrm{x}$-ray laser medium. A theoretical study of the Ni-like Silver x-ray laser has therefore been undertaken to compliment our experimental results, in an attempt to further our understanding of the processes at play in yielding the observed x-ray laser outpul. Preliminary findings are presented within this paper.
\end{abstract}

Keywords: Ni-like, X-ray laser

\section{INTRODUCTION}

Collisional $x$-ray lasers operate in single/double pass, high gain $\left(>10 \mathrm{~cm}^{-1}\right)$, amplified spontaneous emission (ASE) mode where the gain region is an extended plasma column. Population inversion is achieved by collisional excitation of the lasing ion by free electrons. In recent years, a combined experimental and theoretical approach has yielded insights in laser-plasma coupling, refraction and atomic processes influencing gain. This has led to the use of multiple pulse pumping of the lasant material, which has opened the door for saturation to be achieved on a host of XUV lasing lines in the 5.8-60 nm range [1]. Typically, a double pumping pulse arrangement is used where a low intensity prepulse preforms a plasma, which is then irradiated by a high intensity heating pulse to conditions conducive to strong lasing.

Atomic physics codes developed in parallel have allowed for a greater understanding of experimental observations thus opening up further avenues of investigation. Initial development of saturated $\mathrm{x}$-ray lasers focused on multiple $\sim 100 \mathrm{ps}$ pulses with average fluences typically in excess of $20 \mathrm{~J} / \mathrm{cm}$ on target [2]. In this scheme, a quasi-steady state (QSS) population inversion is maintained for $\sim 50 \mathrm{ps}$ until the optimum temperature and density conditions are lost due to plasma expansion and cooling effects. X-ray output duration's of $\sim 40-50 \mathrm{ps}$ have been measured [2].

More recently, theoretical modeling [3] has led to a variation of the QSS scheme in which the main heating of the preformed plasma occurs on a time scale shorter than the relaxation time of the lasing levels. The transient collisional excitation (TCE) scheme utilizes chirped pulse amplification (CPA) technology to rapidly heat the preformed plasma with picosecond pulses. Modeling suggests that a large transient population inversion is obtained via collisional excitation from free electrons on a time scale before ionisation can occur. High gains are expected in the period before collisional redistribution of the excited state populations takes place [4]. It has been postulated that in the TCE scheme the population inversion between the lasing levels in extinguished by over-ionisation of the gain medium [4] however there is a dearth of empirical evidence to support this. In this paper we present preliminary experimental and 
theoretical findings aimed to give insights into the gain dynamics of the Ni-like Ag transient collisional $\mathrm{X}$ ray laser at $139.9 \AA$.

\section{EXPERIMENTAL ARRANGMENT}

In a recent experiment at the Rutherford Appleton Laboratory a fast XUV streak camera with a resolution as high as 1.9 ps was used to diagnose the output of the $J=0 \rightarrow 14 d-4 p$ lasing line 139.9 $[$ [5]. Two beamlines from the Nd-Glass Vulcan laser at $1.06 \mu \mathrm{m}$ were used to irradiate a $10 \mathrm{~mm}$ long flat silver slab target in a standard line focus geometry [6]. A 300ps prepulse typically delivered $10 \mathrm{~J}$ on target into a $21 \mathrm{x}$ $0.12 \mathrm{~mm}$ line focus with intensities of $\sim 2 \times 10^{12} \mathrm{~W} / \mathrm{cm}^{2}$. This pulse generates a plasma which, after a controlled delay, was strongly heated to optimum lasing conditions by a $1.3 \mathrm{ps}$ CPA pulse in a $19 \times 0.08 \mathrm{~mm}$ line focus. Due to the previously inferred [7] short gain durations $(<10 \mathrm{ps})$ relative to the transit time of the $\mathrm{x}$-ray photons with the gain medium $(<30 \mathrm{ps})$ it was necessary to implement traveling wave pumping [6]. It was obscrved that the $\mathrm{x}$-ray lascr cmission pcaked at $\sim 5 \mathrm{mrad}$ off axis for a range of pumping conditions. Through integration of the signal recorded on the CCD we estimate the optimum pumping conditions to be $4.3 \mathrm{~J} / \mathrm{cm}\left(1.2 \times 10^{12} \mathrm{~W} / \mathrm{cm}^{2}\right)$ in the 300 -ps pre-forming pulse and $11.8 \mathrm{~J} / \mathrm{cm}\left(1.1 \times 10^{15} \mathrm{~W} / \mathrm{cm}^{2}\right)$ in the CP $\Lambda$ pulse with the temporal peak-to-peak delay of 200 ps between both pulses. Here, optimum refers to the maximum energy yield within the $\mathrm{x}$-ray laser pulse.

For the time-resolved shots an $\Lambda$ XIS-Photonique XUV streak camera [8] equipped with a $\mathrm{KBr}$ photocathode was positioned at the focal plane of the flat field spectrometer to give temporal resolution of the $\mathrm{x}$-ray laser emission. In our set-up the $1 \times 15 \mathrm{~mm}$ photocathode was placed parallel to the direction of spectral dispersion and at an angular position off axis corresponding to the peak of the $\mathrm{x}$-ray laser output. The streak camera thus gave wavelength resolution at an integrated horizontal angle of $1.1 \mathrm{mrad}$ around the angular peak of the XRL emission. The output from the streak camera was amplified and recorded by the combination of a 50/40 Kentech intensifier butt-coupled to an optical CCD camera. This configuration was estimated to give a temporal resolution of $1.92 \pm 0.16 \mathrm{ps}$. Figure 1(a) shows the time resolved spectral output from the Ni-like Ag emitted at the optimal irradiation conditions in the traveling wave geometry, as recorded at the output of the streak camera. The cut-off of the $\mathrm{x}$-ray laser output is observed to coincide with the increased continuum emission by free electrons. Figure 1(b) shows the wavelength-integrated output of the $\mathrm{x}$-ray laser as a function of time after the continuum emission has been subtracted. The FWHM duration of the $\mathrm{x}$-ray laser was measured to be $1.8 \pm 1.0 \mathrm{ps}$.

It may be seen in figures 1 (a) and (b) that the $\mathrm{x}$-ray signal was extinguished on the rising edge of the continuum emission. This observation was repeatable for a number of different pump laser parameters. One possible interpretation of this result is that the rising edge of the continuum emission is symptomatic of over-ionisation within the plasma which in turn signals the extinction of the population inversion on the gain line. One may also postulate, however, that the relative time delay between the peak $\mathrm{x}$-ray laser output and the peak continuum emission is a result of a thermal heat wave traveling towards the dense target surface. Such an effect would not necessarily impact on the population dynamics in the lasing ion. The nature of our experimental setup meant that no information regarding the spatial distribution of the continuum emission in relation to the $\mathrm{x}$-ray laser output was available. In addition, we took a number of shots were we changed the laser parameters (main heating pulse duration and long pulse/short pulse separation) and witnessed a two fold increase in x-ray laser duration. The number of data points at these conditions was not sufficiently high to conclusively point to a definite effect but further investigation is warranted.

This high-resolution characterization of the $\mathrm{x}$-ray laser output yielded previously unobtainable insights into the nature of the $\mathrm{x}$-ray laser output for the transient collisional excitation scheme. It is clear, however, that to fully explain these observations a comprehensive theoretical modeling effort is needed to compliment the experimental work. 

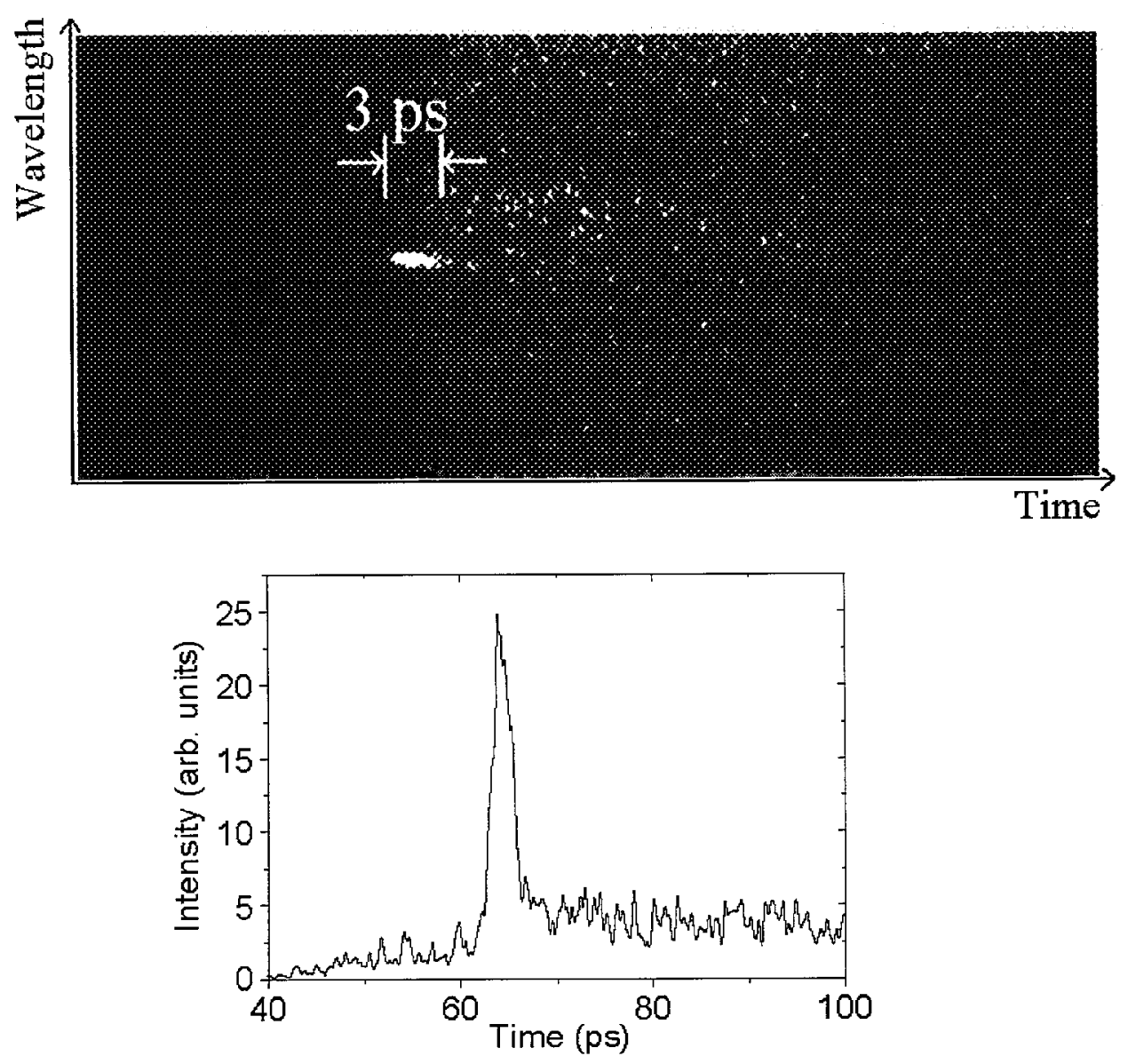

Figure 1. Time resolved spectrum of the Ni-like transient pumping silver $\mathrm{x}$-ray laser at the optimal traveling wave irradiation conditions. Figure 1(a) represents the time resolved output of the Ni-like Ag x-ray laser as recorded by a CCD camera. Figure $1(\mathrm{~b})$ shows the intensity versus time plot at the lasing wavelength. The FWHM x-ray laser duration at $139.9 \AA$ was measured to be $1.8 \pm 1.0 \mathrm{ps}$. The irradiation conditions are: $4.3 \mathrm{~J} / \mathrm{cm}\left(1.2 \times 10^{12} \mathrm{~W} / \mathrm{cm}^{2}\right)$ in the 300 -ps pre-forming pulse and $11.8 \mathrm{~J} / \mathrm{cm}\left(1.1 \times 10^{15} \mathrm{~W} / \mathrm{cm}^{2}\right)$ in the 1.3 -ps CPA pulse with the temporal peak-to-peak delay of 200 ps between both pump laser pulses.

\section{THEORETICAL MODELING}

Simulations of the experimental conditions described above were carried out using the EHYBRID 1.5D hydrodynamic atomic physics code developed at the University of York in the UK [9]. EHYBRID which was developed to understand the evolution of the lasing material and describes many physical processes, including pump laser energy deposition, hydrodynamical motion, electron thermal conduction, ion-electron thermalisation coupled with the atomic physics of the lasant [10]. The calculation of the ionisation balance within the plasma, and in particular the abundance of the Ni-like ions is of central importance in these simulations. In EHYBRID the time dependant ionisation is calculated using a collisional radiative model [10].

The input to the Ehybrid code is a file containing the atomic data of the lasing material and a description of the experimental pumping laser parameters. Absorption of the pumping laser is assumed to be by inverse Bremsstrahlung (IB) with refraction considered. The IB coefficient is calculated for the propagation of the laser pulse towards the target surface and as the laser pulse is reflected away from the critical surface. High field modifications of this coefficient are not included. Resonant absorption is modeled by assuming a $30 \%$ 
dump of the laser energy reaching the critical surface. Collisional excitation, de-excitation, radiative decay, collisional ionisation, three-body recombination, radiative recombination processes are all included in the coupled rate equation solution for each level. The ionisation rate approximation of Golden et al. [11] is implemented, with three-body recombination calculated from a detailed balance inverse [10]. The code uses a 98 Lagrangian cell matrix in the direction away from the target with the output file consisting of various calculated plasma parameters (Electron temperature, Density, Gain, Ionisation etc.) given as a function of cell position and time. The fluid is modeled in the direction parallel to the driving laser by cells which are assumed to be laterally isothermal and, as such, the transverse expansion is considered to be selfsimilar [9]. To fully compare simulation with experiment the output of the code is coupled to a raytracing post-processor which models the transport of the $\mathrm{x}$-ray laser along the extended plasma column [12]. The raytracing code takes into account effects due to refraction and saturation and can operate in a traveling irradiation wave geometry of $\mathrm{v}=\mathrm{c}$ or $\infty$, where $\mathrm{v}$ is the velocity of the traveling wave. EHYBRID has shown to give excellent agreement to experimental results for the Ne-like Ge $\mathrm{x}$-ray laser [17].

The atomic physics input file for $\Lambda \mathrm{g}$ was constructed at LSAI, in Orsay. The Ni-like ion stage is modelled with 272 excited levels including all levels in the $\mathrm{n}=4$ and 5 manifolds and with averaged contributions from the $n=6$ to 8 levels. Ion stages other than the Ni-like are treated with varying degrees of complexity using a screened hydrogenic model or a simpler two-level model based on the modified form of the Griems model [13]. Electron-Ion collisional cross-sections were calculated at LULI (Ecole Polytechnique) for all transitions within the $\mathrm{n}=4$ manifold. Oscillator Strengths for all transitions in the $\mathrm{n}=4$ to 8 manifolds were calculated with a multi-configurational Dirac-Fock code (MCDF) [14]. In all of these calculations the $4 p$ and $4 s$ shells are left open.

\section{RESULTS}

The input conditions to the simulations were set to model those which gave the x-ray laser output shown in figure 1. Figure 2 shows the gain on the primary line for Ni-like $\mathrm{Ag}$ on the $4 \mathrm{~d}-4 \mathrm{p}{ }^{1} \mathrm{~S}_{0} \rightarrow{ }^{1} \mathrm{P}_{1}$ lasing transition at $139.9 \AA$ as a function of time and distance away from the target surface. The peak of the 1.3ps FWHM heating pulse occurs at $0.65 \mathrm{ps}$ on the time scale. There are two distinct regions predicted. At earlier times when the 1.3ps heating pulse is turned on there is an emergence of a large $\left(>500 \mathrm{~cm}^{-1}\right)$ gain region with small dimensions $(<5 \mu \mathrm{m})$ within $15 \mu \mathrm{m}$ of the target surface. When the laser is turned off and the plasma expands a larger gain plateau with gains $<400 \mathrm{~cm}^{-1}$ extending out to $\sim 40 \mu \mathrm{m}$ is observed. To what extent these gain regions contribute to the $\mathrm{x}$-ray laser output is largely dependent on the density scale lengths which exist within the plasma. The smaller the density scale lengths at a given point in space the more refraction will tend to preclude spatial sampling by the $\mathrm{x}$-ray beam as it propagates along the line focus. Figure 3 shows the predicted temporal evolution of the electron density profile as a function of distance away from the target surface. On comparison with the predicted evolution of the local gain we see that the high gain peaks at electron densities close to $10^{21} \mathrm{~cm}^{-3}$. This is the critical density surface of the $1.06 \mu \mathrm{m}$ laser driver and coincides spatially with a region of steep density gradients. It would be expected, therefore, that spatially sampling of this region would be limited by refraction. It is observed that the density gradients become more relaxed at distances away from the critical density surface and so due to reduced refraction effects and its larger dimensions the larger lower gain region is expected to contribute most to the $\mathrm{x}$-ray laser output. 


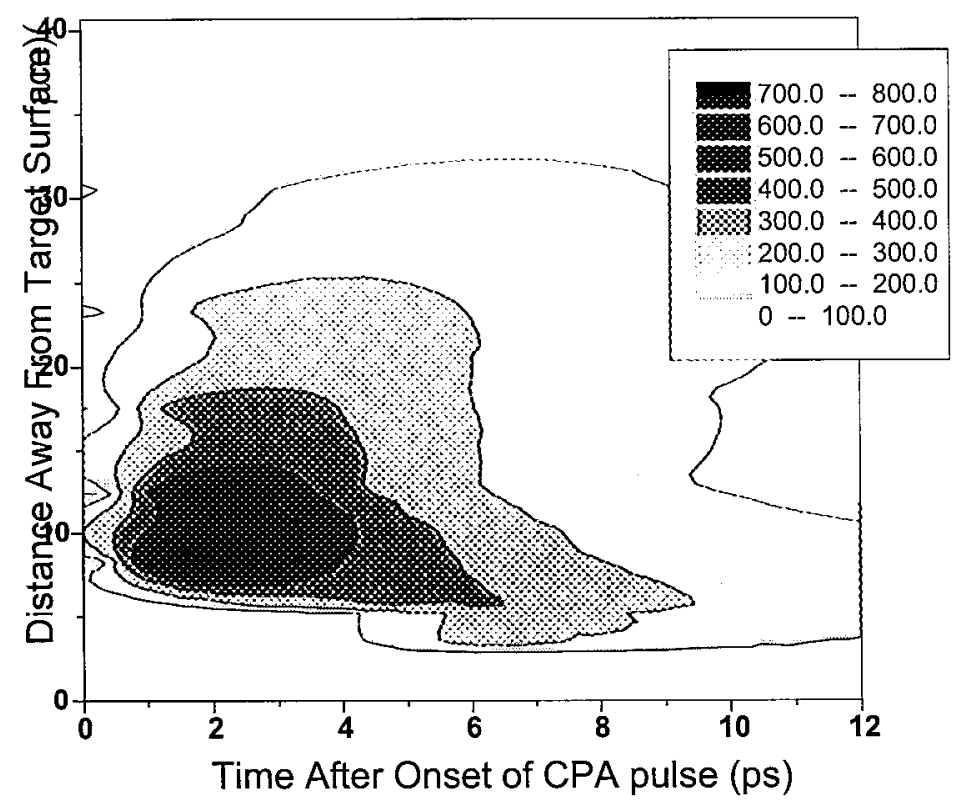

Figure 2: Local gain on the $4 \mathrm{~d}-4 \mathrm{p}{ }^{1} \mathrm{~S}_{0} \rightarrow{ }^{1} \mathrm{P}_{1}$ lasing transition as a function of time and distance and time away from target surface. The peak of the $1.3 \mathrm{ps}$ FWHM CPA pulse occurs at $0.65 \mathrm{ps}$.

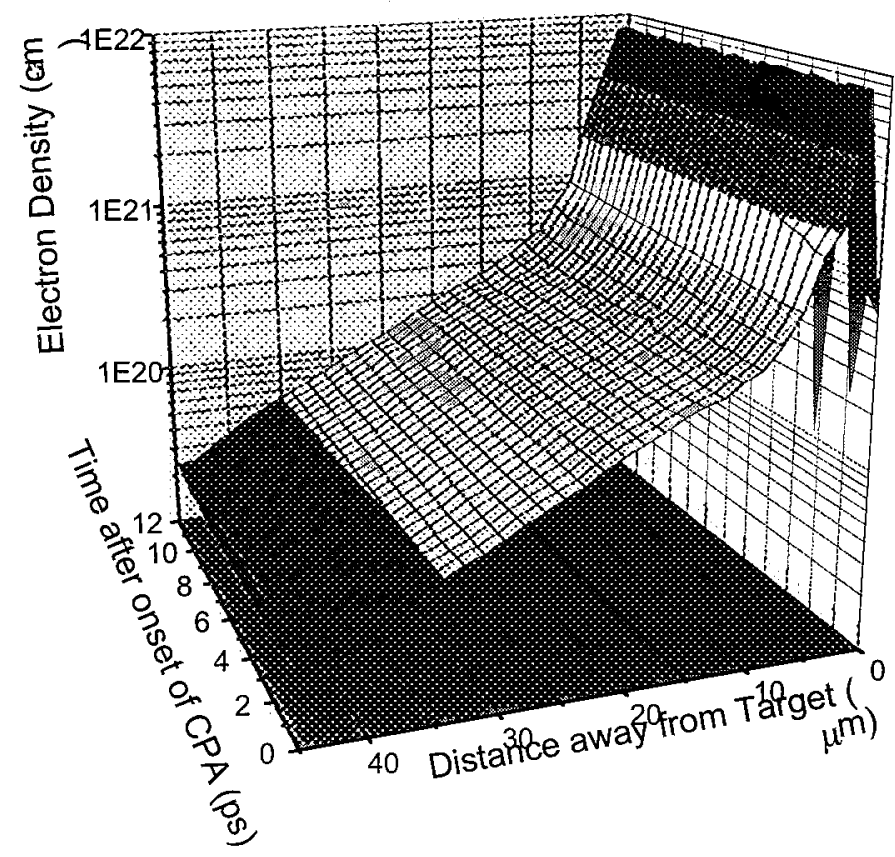

Figure 3: Temporal evolution of the free electron density as a function of time and distance away from the target surface. High gain peak predicted in figure 2 is shown to reside at below $15 \mu \mathrm{m}$ from the target were steep density gradients and the associated refractive effects would prohibit extensive sampling by the $\mathrm{x}$-ray laser beam.

In figure 4 the temporal evolution of the electron temperature as a function of distance away from the target surface is shown. Temperatures reaching $1800 \mathrm{eV}$ are expected within the critical density surface. The temperature falls away quickly at distances further away from the target. A trend similar to that of the gain 
profile in figure 2. At times after the main heating pulse is turned off the bulk of the plasma stays hot $(>500 \mathrm{eV})$ for several picoseconds. This prolonged heating causes the average ionisation of the plasma to increase rapidly. This effect is illustrated in figure 5 were the average ionisation is given for each of the 98 Lagrangian cells as a function of time. In the region of space-time where the high gains are predicted to occur the average ionisation is less than the $\mathrm{Z}=19$ associated with the Ni-like stage. However the fact that such high gains are predicted here is indicative of the large densities in this supra-critical region. It is observed that after a short time $(\sim \mathrm{ps})$ the plasma over-ionises. Previous work has attributed this effect and the associated collisional redistribution of the excited level populations to be the main mechanism for extinguishing the population inversion on the gain transition [4]. Such a hot, ionizing plasma with consistent densities would be expected to have enhanced continuum emission as observed in figure one. Fulure work will concentrate on spatially simulating this emission in the timc domain to further compare to experiment. The calculations described in figures $2,3,4$ and 5 are very similar to those predicted by other hydrodynamic codes for $\mathrm{Ag}[15]$.

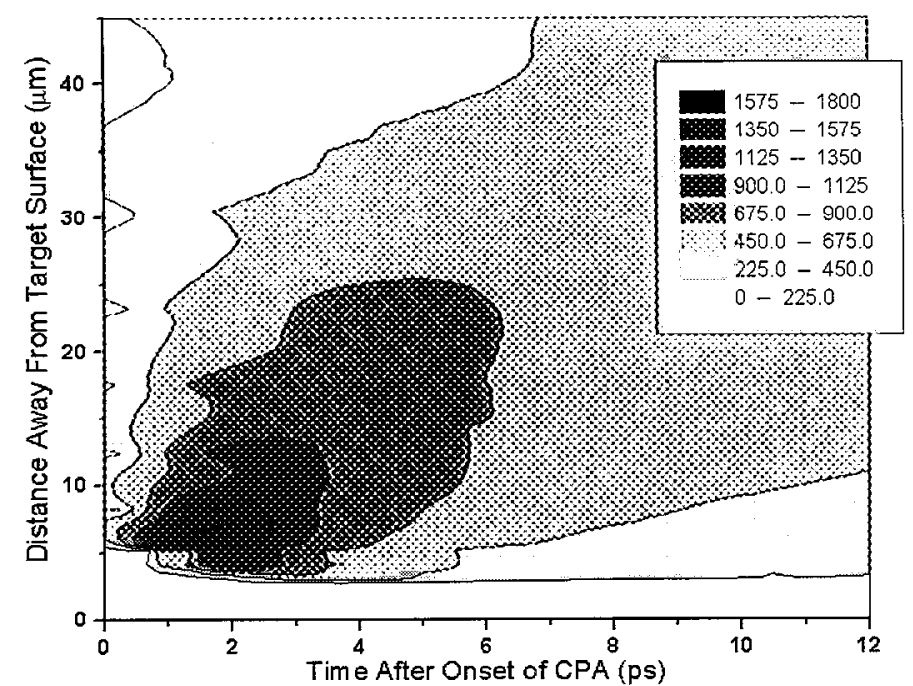

Figure 4: Heating of the super-critical densities to temperatures up to $1.8 \mathrm{keV}$ is predicted during the main heating pulse. These greater than $1 \mathrm{keV}$ temperatures co-exist spatially and temporally with the gain spike shown in figure 2 . The temperature gradients are similar to those witnessed for the gain with quick dissipation from a small intense region into a larger more uniform plateau with temperatures below $500 \mathrm{eV}$.

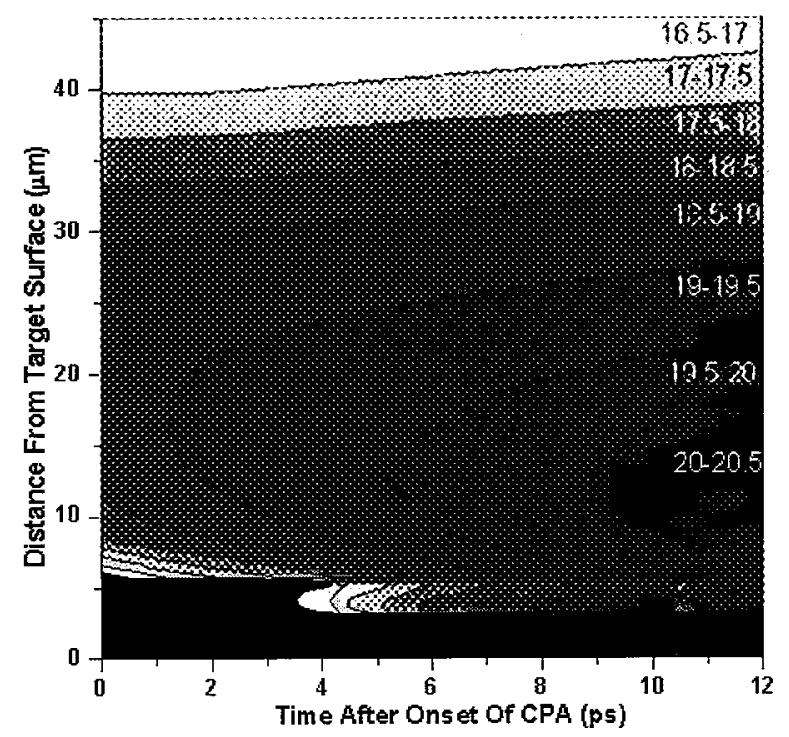

Figure 5: Temporally resolved average ionisation within each of the 98 Lagrangian cells expanding away from the target surface. 
In order to compare the output of EHYBRID with experimental observations it was necessary to use a raytracing post-processor [12]. The raytracing code models the refraction of the $\mathrm{x}$-ray laser pulse as it propagates through the gain medium. Saturation effects are taken into consideration. In figure 6 the predicted output of the Ni-like x-ray laser is shown. Such an output is directly comparable to that shown in figure 1. The plot illustrates the predicted output of the $\mathrm{x}$-ray laser in angular space perpendicular to the target surface and as a function of time. The deflection angle of $\sim 6 \mathrm{mrad}$ is in good agreement with the $\sim 5 \mathrm{mrad}$ measured experimentally. The expected FWHM of the output pulse is $0.14 \mathrm{ps}$ compared to the experimental value of $1.8 \pm 1 \mathrm{ps}$. However it is noticed that from the colour table associated with this plot that the intensity peak of the $\mathrm{x}$-ray laser output is extremely high. The small signal gain predicted by the raytracing code in $\sim 500 \mathrm{~cm}^{-1}$. Clearly this is a large discrepancy from the measured values of $-35 \mathrm{~cm}^{-1}$. Further analysis of the predicted $\mathrm{x}$-ray output as a function of length suggests that there may be inaccuracics in the way saturation is applied to the $x$-ray laser during transit thus leading to unrealistic output intensity units. Future work will address this issue.

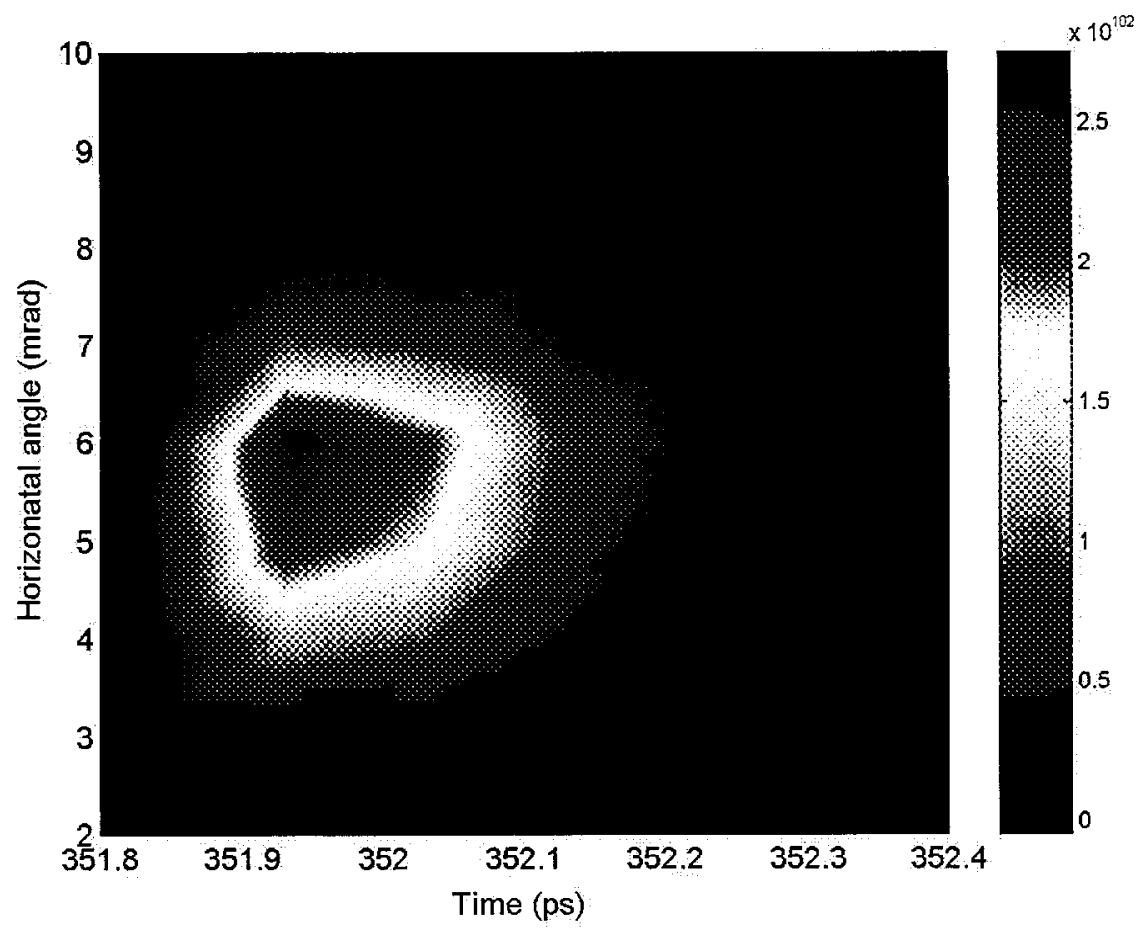

Figure 6: Predicted output of the $\mathrm{x}$-ray laser as a function of angle away from the target surface and time after the onset of the $1.3 \mathrm{ps}$ heating pulse. The heating pulse turns on at $350 \mathrm{ps}$ and peaks at $350.65 \mathrm{ps}$.

\section{DISCUSSION}

Ehybrid simulations coupled with raytracing post-processing have previously shown excellent agreement to experimental observations for the quasi-steady state 100ps pumping scheme [16]. In this scheme any nonuniformities associated with the pumping laser and target roughness have time to coalesce and smooth out during the main heating pulse. Therefore, even though the modeling described above does not take into account any such non-uniformities no large discrepancies are observed with experiment for the QSS scheme because of the time scales involved. There are two main reasons why the transient pumping scheme is less forgiving and more likely to accentuate differences between theory and experiment. Firstly, the short picosecond time scales involved in the traveling wave heating and gain generation mcans that the inhomogeneities within the plasma are fully sampled by the $x$-ray laser pulse during transit along the line focus. This is observed in near-field imaging experiments where detailed mode structure is clearly visible at the output plane of the gain medium [16]. Such results clearly indicate that the smoothly evolving gain profile predicted in figure 2 is not experimentally realized. Secondly, the gain medium for the transient 
scheme lies closer to the target surface and in regions of steeper density gradients than that for the $100 \mathrm{ps}$ pumping schemes. Predicted outputs are therefore more sensitive to the accuracy of the raytracing calculations.

\section{CONCLUSIONS}

Experimental observations have shown the transient $\mathrm{Ni}$-like $\mathrm{Ag}$ x-ray laser to have a duration $\sim 1.8 \mathrm{ps}$ under optimum pumping conditions at RAL. The time resolved output of the $\mathrm{x}$-ray laser plasma has consistently shown that lasing is extinguished on rising edge of continuum. One possible explanation is that this increased emission is symptomatic of over-ionisation within the plasma. And this, as previously, suggested is the main mechanism for destroying the population inversion. In addition, preliminary experimental results suggested that the duration was also sensitive on laser pump parameters. An atomic data file was constructed for the atomic physics code Ehybrid in order to better understand population and plasma dynamics. High gains $\left(-700 \mathrm{~cm}^{-1}\right)$ were predicted near critical surface with broad lower gain $\left(\sim 200 \mathrm{~cm}^{-1}\right)$ plateau at sub-critical densities. The plasma expected to over-ionise after $\sim 5-10$ picoseconds. Subsequent raytracing predicted the deflection angle to be $\sim 5 \mathrm{mrad}$ which was close to experimental observations with the peak out occurring at $\sim 1.9 \mathrm{ps}$ after the onset of the main 1.3ps heating pulse. The duration of the $\mathrm{x}$-ray laser is expected to be $0.14 \mathrm{ps}$ compared to a measured value of $1.8 \pm 1 \mathrm{ps}$ with the resolution of the experimental setup at $1.9 \mathrm{ps}$. Large discrepancies exist in the raytraced output and the associated small signal gain to those measured in the lab. It is suggested that plasma non-uniformities over the short picosecond time scales coupled with the traveling wave pumping geometry contribute to this offset.

\section{ACKNOWLEDGEMENTS}

The authors would like to thank Joseph Nilsen at LLNL for helpful discussions in preparing this paper.

R. Smith was funded by the TMR x-ray laser network contract \#ERBFMRXCT98-0185.

\section{REFERENCES}

1. R. Smith, G.J. Tallents, J. Zhang, G. Eker, S. McCahe, G.I. Pert \& E. Wolfrum, Phys. Rev. A 59, R47 (1999); R. Tommasini, F. Lowenthal \& J.E. Balmer, Phys. Rev. A, 59 (2), 1577 (1999); F.G. Tomasel, J.J. Rocca, V.N. Shlyaptsev \& C.D. Macchietto, Phys. Rev. A 55, 1437 (1997).

2. J. Zhang, A.G. MacPhee, J. Lin, E.Wolfrum, R. Smith, C. Danson, M.H. Key, C.L.S. Lewis, D. Neely, J. Nilsen, G.J. Pert, G.J. Tallents \& J.S. Wark, Science 276, 1097 (1997).

3. Yu.V. Afanasiev and V.N. Shlyapsev, Sov. J. Quantum Electronics. 19, 1606 (1989); V.N. Shlyaptsev et al., Proc. SPIE Int. Soc. Opt. Eng. 2012, 111 (1993).

4. J. Nilsen, Yuelin Li, J. Dunn, J. Opt. Soc. Am B, 17 (6), (2000).

5. A. Klisnick, J. Kuba, D. Ros, R. Smith, G. Jamelot, C. Chenais-Popovics, R. Keenan, S. Topping, C.L.S. Lewis, F. Strati, G.J. Tallents, D. Neely, R. Clarke, J. Collier, A.G. MacPhee, F. Bortolotto, K.A. Janulewicz, P.V. Nickles, submitted to Phys. Rev. A (2001); A. Klisnick et al., these proceedings.

6. G.J. Tallents et al., App. of High Field and Short Wavelength Sources VIII OSA Tech. Digest Series 7 (47), (1997); M.P. Kalachnikov et al., Phys. Rev. A, 57 (6), 4778 (1998); C.L.S. Lewis et al., SPIE $3776(1999)$.

7. J. Kuba, A. Klisnick, D. Ros, P. Fourcade, G. Jamelot, J.-L. Miquel, N. Blanchot, J.-F. Wyart, Phys. Rev. A.62 (4), p.43808 (2000).

8. Cote et al., Proc. SPIE 2869, p. 956-961, 22nd International Congress on High-Speed Photography and Photonics, Dennis I. Paisley Fd.

9. G.J. Pert J. Fluid. Mech. 131, 401 (1983).

10. S.B. Healy et al., Phys. B: At. Mol. Opt. Phys. 28, 1381-1391 (1995); P.B. Holden et al., J. Phys. B: At. Mol. Opt. Phys. 27, 341-367 (1994); S.B. Healy et al., IEEE J. of Selected Topics in Quant. Elect., 1 (3), 949 (1995).

11. L.B. Golden, R.E.H. Clark, S.J. Goett and D.H. Sampson, Astrophys. J. Suppl. 45, 603 (1981).

12. J.A. Plowes, G.J. Pert, S.B. Healy, and D.T. Toft, Optical and Quantum Electronics 28, 219 (1996); J.A. Plowes, G.J. Pert, and P.B. Holden, Opt. Comm. 116, 260 (1995). 
13. H. Griem, Plasma Spectroscopy, MacGraw Hill, New York 1964.

14. I.P. Grant, Adv. Phys. 19, 747 (1970).

15. J. Nilsen, these proceedings.

16. J. Dunn et al., these proceedings.

17. J.Y. Lin, G.J. Tallents, A. Demir, S.B. Healy, and G.J. Pert, J. Applied Phys. 83, 1863 (1998).

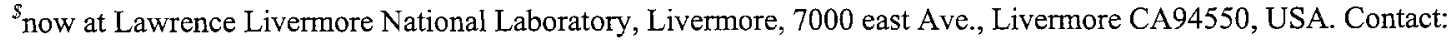
smith248@11nl.gov 